\title{
Can we exonerate GLP-1 receptor agonists from blame for adverse pancreatic events?
}

\author{
Conor F. Murphy ${ }^{1}$, Carel W. le Roux ${ }^{1,2}$ \\ ${ }^{1}$ Diabetes Complications Research Centre, Conway Institute, University College Dublin, Dublin, Ireland; ${ }^{2}$ Gastrosurgical laboratory, Sahlgrenska \\ Academy, University of Gothenburg, Gothenburg, Sweden \\ Correspondence to: Professor Carel W. le Roux. Diabetes Complications Research Centre, Conway Institute, University College Dublin, Dublin, \\ Ireland. Email: carel.leroux@ucd.ie. \\ Provenance: This is a Guest Editorial commissioned by Section Editor Kaiping Zhang (AME College, AME Group, Hangzhou, China) and Guest \\ Section Editor Hengrui Liang (Nanshan Clinical Medicine School, Guangzhou Medical University, Guangzhou, China). \\ Comment on: Steinberg WM, Buse JB, Ghorbani MLM, et al. Amylase, Lipase, and Acute Pancreatitis in People With Type 2 Diabetes Treated With \\ Liraglutide: Results From the LEADER Randomized Trial. Diabetes Care 2017;40:966-72.
}

Submitted Feb 09, 2018. Accepted for publication Feb 25, 2018.

doi: $10.21037 / \mathrm{atm} .2018 .03 .06$

View this article at: http://dx.doi.org/10.21037/atm.2018.03.06

Several randomised controlled trials (RCTs) have proven the efficacy of glucagon-like peptide-1 (GLP-1) receptor agonists (GLP-1RA), such as liraglutide, in both improving glycaemic control, inducing sustained significant weight loss, and reducing mortality in patients with diabetes. These benefits have been demonstrated in myriad cohorts, as patients with type 2 diabetes (T2D), prediabetes and normoglycemia all exhibit positive outcomes (1-4).

With widespread localisation of GLP-1 receptors, both centrally and peripherally (5), incretin-based therapies capitalise on the pleiotropic effects of GLP-1 stimulation. For example, to induce weight loss, GLP-1RA create an anorexigenic balance between feelings of hunger and satiation through activation of neurones residing in the arcuate nucleus of the hypothalamus and hind brain (6), yet it is via stimulation of the alpha cell GLP-1 receptors in the pancreas that it exerts its glycaemic benefits, taking advantage of the incretin effect (7).

In light of this pleiotropy, many discussions have taken place with regards to potential adverse pancreatic and extra-pancreatic effects of GLP-1RA, such as acute and chronic pancreatitis, pancreatic cancer, mild tachycardia, and thyroid cancer $(8,9)$, and indeed its major side effects do include gastrointestinal symptoms, in keeping with its recognised gut motility-altering properties (10). However, evidence exploring the adverse effects of stimulating its extra-pancreatic targets have remained largely inconclusive, with risk of thyroid carcinoma limited to murine models (9), and any potential harm arising as a result of tachycardia has lacked a robust mechanistic basis (8).

Whether pharmacological exploitation of enteroendocrine physiology is to blame for adverse pancreatic events observed during treatment with GLP$1 \mathrm{RA}$ has been a contentious issue, complicated by conflicting evidence, and diverse opinions (11-15). Murine and preclinical models have demonstrated the potential of GLP-1RA to induce histopathological changes in both the exocrine and endocrine pancreatic components, with dysplasia and chronic inflammation in the former, and hyperplasia in the latter $(16,17)$. Overall, given the presence of mechanistic and observational evidence suggesting an association between adverse pancreatic events and GLP1RA, understandable concerns have been raised. Although, admittedly, this is in the context of relatively weak evidence, subject to risks of methodological limitations such as confounding by indication and time-lag bias. That being said, more recent meta-analytical evidence appears to dilute the notion of a link between acute pancreatitis and GLP1RA therapy, albeit specifically in patients with T2D $(18,19)$.

Steinberg et al., in Diabetes Care, recently made a valuable contribution to this realm of the literature. They present a comprehensive evaluation of the comparative trends of both serum amylase and lipase, as well as how they relate to the rates of acute pancreatitis observed in patients with T2D 
receiving either liraglutide therapy, or placebo (20). As part of the LEADER RCT of cardiovascular outcomes (4), data were collected detailing pancreatic events in 9,340 patients exposed to treatment, and observed, for medians of 3.52 and 3.84 years, respectively.

While the liraglutide cohort demonstrated increases in serum lipase and amylase at 36 months of follow-up (28.0\% and $7.0 \%$, respectively) relative to placebo, there was no significant difference in the incidence of acute pancreatitis between groups. Eighteen of the 4,668 patients treated with liraglutide $(0.4 \%)$, and 23 of $4,672(0.5 \%)$ receiving placebo, developed acute pancreatitis [hazard ratio (HR), 0.78; $95 \%$ CI, 0.42-1.44], the diagnosis of which was discerned by independent and blinded adjudication. In addition, the aforementioned rises in pancreatic enzymes did not predict the risk of subsequent acute pancreatitis, with a positive predictive value of $<1.0 \%$ in liraglutide-treated patients.

The vast majority of cases of pancreatitis in both groups were mild (liraglutide: $89.5 \%$ vs. placebo: $83.9 \%$ ), and most occurred at least 12 months after randomisation. Moreover, one third of those afflicted in the liraglutide group had been off treatment for 28-637 days. The authors note that, although liraglutide therapy in this cohort was shown to increase the risk of gallstones and acute cholecystitis (4), there was no difference in the incidence of gallstonerelated episodes of acute pancreatitis (liraglutide: $38.9 \% v s$. placebo: $43.5 \%$ ). Lastly, while $5 \%$ of those having an event in the placebo group had a history of acute pancreatitis, this was the case for only $1.9 \%$ of the liraglutide group, but this risk factor did not emerge as a significant predictor, when analysed by logistic regression ( $\mathrm{P}=0.13)$.

As emphasized by the authors, this study has several strengths, in particular its large cohort, in combination with the randomised, double-blind study design. In addition, the prospective collection of adverse event data, incorporating blinded adjudication of acute pancreatitis based on prespecified standardised criteria, with its severity based on the revised Atlanta criteria, enhances the robustness of the reported results. Notwithstanding, it must be highlighted, given the indications for use of GLP-1RA in, for example, obese patients without T2D, or with prediabetes, generalizability of this study is relatively limited. Its sample draws from only those with T2D, of mean duration 12.9 years, at high cardiovascular risk. The authors also concede that the low number of events limit their ability to definitively refute the presence of an elevated risk of acute pancreatitis. Finally, it must be borne in mind that T2D is a confounding risk factor for acute pancreatitis, and a higher proportion of liraglutide-treated patients had shorter duration of T2D when dichotomised using a 11-year cutoff (liraglutide vs. placebo, $\leq 11$ years; HR, 0.82; 95\% CI, 0.70-0.97). Neither baseline T2D duration, nor baseline glycated haemoglobin, however, interacted significantly with subsequent lipase or amylase levels between treatment cohorts.

Interestingly, this study calls into question the requirement of pancreatic enzyme monitoring with respect to predicting acute pancreatitis. With the positive predictive value set at $<1.0 \%$, a cost-benefit analysis would almost certainly fall short of advocating for continuation of this practice in symptomfree patients. Furthermore, the current warning labels of adverse pancreatic events are becoming less warranted as more evidence is presented. This is particularly the case for liraglutide treatment, which does not appear to serve as a cumulative risk factor, with a history of acute pancreatitis, for an acute adverse pancreatic event. Nevertheless, the underlying explanations for these enzymatic elevations remain elusive, although several postulated mechanisms exist, such as an increased basolateral secretion of pancreatic enzymes from the exocrine acinar cells, and possible alterations in the elimination of lipase, a renally excreted compound (21).

On reflection, the observation that one in three of those developing acute pancreatitis in the liraglutide cohort did so while off treatment, may be interpreted from antagonistic perspectives. It could be of concern should a link between incretin therapy and adverse pancreatic events become conclusive, as it might suggest a lasting adverse effect of treatment beyond cessation. On the other hand, it could be interpreted as a reason to disassociate GLP-1RA from acute pancreatitis, justified by the fact that patients weren't taking the medication at time of diagnosis. When contemplating the emergence of acute pancreatitis while on treatment, perhaps as a differentiating factor between the impact of active and comparative treatment, one might suspect a relatively stable time-course of incident acute pancreatitis over a prolonged period of time in those receiving placebo. Yet, it actually mirrored that of liraglutide-treated patients insofar as most cases occurred after one year of treatment in both groups. However, the low event rate may limit the ability to observe such a difference.

Importantly, while acute pancreatitis represents a painful and potentially life-threatening condition, many would argue that in the face of such low excess risk, whether there is a relationship or not with GLP-1RA, their use would not be precluded given their consistent therapeutic benefits. Indeed, perhaps an outcome of more relevance 
is the potential relationship with pancreatic cancer risk, although this connection is also disputed, and controversial $(22,23)$. Alluding to this topic, the LEADER investigators appropriately noted that while there was a non-significant increase in adverse event rate with liraglutide $(0.3 \% v s$. $0.1 \%$ with placebo, $\mathrm{P}=0.06$ ), the study was not powered to detect cancer risk and thus could not make a conclusive statement. This is an issue that most certainly requires further dedicated investigation, with long-term follow-up, for example as part of post-marketing surveillance.

To summarise, Steinberg et al. put forward reassuring evidence indicating that the addition of liraglutide therapy should not be a cause for concern, from the perspective of acute pancreatitis, for the majority of those with T2D at high cardiovascular risk. Pancreatic enzymes appear fruitless as predictive markers of acute pancreatitis, but the longterm pancreatic consequences of their liraglutide-induced elevations are unclear and require further investigation. However, while these findings represent a meaningful contribution to the debate regarding acute pancreatitis, it would be premature to grant the exoneration of liraglutide from having a causative role in any adverse pancreatic events. Future research in patients with T2D taking GLP1RA is necessary to ascertain a definitive answer on issues such as pancreatic cancer, which would require longerterm follow-up, appropriately powered studies, and histopathological evidence. With the success of recent trials, rates of therapy with GLP-1RA will continue to rise providing opportunities to categorically elucidate their true risk-benefit profile, allowing evidence-based and unambiguous discourse between physician and patient.

\section{Acknowledgements}

Funding: This work was supported by a scholarship award from the Irish Cancer Society, Ireland, to Dr. Conor F. Murphy (CRS17MUR).

\section{Footnote}

Conflicts of Interest: CW le Roux serves on advisory boards for NovoNordisk, Johnson \& Johnson, GI Dynamics, Fractyl and Herbalife. CF Murphy has no conflicts of interest to declare.

\section{References}

1. le Roux CW, Astrup A, Fujioka K, et al. 3 years of liraglutide versus placebo for type 2 diabetes risk reduction and weight management in individuals with prediabetes: a randomised, double-blind trial. Lancet 2017;389:1399-409.

2. Pi-Sunyer X, Astrup A, Fujioka K, et al. A Randomized, Controlled Trial of $3.0 \mathrm{mg}$ of Liraglutide in Weight Management. N Engl J Med 2015;373:11-22.

3. Potts JE, Gray LJ, Brady EM, et al. The Effect of Glucagon-Like Peptide 1 Receptor Agonists on Weight Loss in Type 2 Diabetes: A Systematic Review and Mixed Treatment Comparison Meta-Analysis. PLoS One 2015;10:e0126769.

4. Marso SP, Daniels GH, Brown-Frandsen K, et al. Liraglutide and Cardiovascular Outcomes in Type 2 Diabetes. N Engl J Med 2016;375:311-22.

5. Pyke C, Heller RS, Kirk RK, et al. GLP-1 receptor localization in monkey and human tissue: novel distribution revealed with extensively validated monoclonal antibody. Endocrinology 2014;155:1280-90.

6. Secher A, Jelsing J, Baquero AF, et al. The arcuate nucleus mediates GLP-1 receptor agonist liraglutide-dependent weight loss. J Clin Invest 2014;124:4473-88.

7. Meloni AR, DeYoung MB, Lowe C, et al. GLP-1 receptor activated insulin secretion from pancreatic $\beta$-cells: mechanism and glucose dependence. Diabetes Obes Metab 2013;15:15-27.

8. Nauck MA. A critical analysis of the clinical use of incretin-based therapies: The benefits by far outweigh the potential risks. Diabetes Care 2013;36:2126-32.

9. Chiu WY, Shih SR, Tseng CH. A review on the association between glucagon-like peptide-1 receptor agonists and thyroid cancer. Exp Diabetes Res 2012;2012:924168.

10. Keller J, Trautmann ME, Haber H, et al. Effect of exenatide on cholecystokinin-induced gallbladder emptying in fasting healthy subjects. Regul Pept 2012;179:77-83.

11. Jensen TM, Saha K, Steinberg WM. Is there a link between liraglutide and pancreatitis? A post hoc review of pooled and patient-level data from completed liraglutide type 2 diabetes clinical trials. Diabetes Care 2015;38:1058-66.

12. Thomsen RW, Pedersen L, Møller N, et al. Incretinbased therapy and risk of acute pancreatitis: a nationwide population-based case-control study. Diabetes Care 2015;38:1089-98.

13. Azoulay L. Incretin-based drugs and adverse pancreatic events: almost a decade later and uncertainty remains. Diabetes Care 2015;38:951-3.

14. Li L, Shen J, Bala MM, et al. Incretin treatment and risk of pancreatitis in patients with type 2 diabetes mellitus: 
systematic review and meta-analysis of randomised and non-randomised studies. BMJ 2014;348:g2366.

15. Butler PC, Elashoff M, Elashoff R, et al. A critical analysis of the clinical use of incretin-based therapies: Are the GLP-1 therapies safe? Diabetes Care 2013;36:2118-25.

16. Butler AE, Campbell-Thompson M, Gurlo T, et al. Marked expansion of exocrine and endocrine pancreas with incretin therapy in humans with increased exocrine pancreas dysplasia and the potential for glucagon-producing neuroendocrine tumors. Diabetes 2013;62:2595-604.

17. Gier B, Matveyenko AV, Kirakossian D, et al. Chronic GLP-1 receptor activation by exendin-4 induces expansion of pancreatic duct glands in rats and accelerates formation of dysplastic lesions and chronic pancreatitis in the Kras(G12D) mouse model. Diabetes 2012;61:1250-62.

18. Monami M, Nreu B, Scatena A, et al. Safety issues with glucagon-like peptide-1 receptor agonists (pancreatitis, pancreatic cancer and cholelithiasis): Data from randomized controlled trials. Diabetes Obes Metab

Cite this article as: Murphy CF, le Roux CW. Can we exonerate GLP-1 receptor agonists from blame for adverse pancreatic events? Ann Transl Med 2018;6(10):186. doi: 10.21037/ atm.2018.03.06
2017;19:1233-41.

19. Storgaard H, Cold F, Gluud LL, et al. Glucagon-like peptide-1 receptor agonists and risk of acute pancreatitis in patients with type 2 diabetes. Diabetes Obes Metab 2017;19:906-8.

20. Steinberg WM, Buse JB, Ghorbani MLM, et al. Amylase, Lipase, and Acute Pancreatitis in People With Type 2 Diabetes Treated With Liraglutide: Results From the LEADER Randomized Trial. Diabetes Care 2017;40:966-72.

21. Junge W, Mályusz M, Ehrens HJ. The role of the kidney in the elimination of pancreatic lipase and amylase from blood. J Clin Chem Clin Biochem 1985;23:387-92.

22. Azoulay L, Filion KB, Platt RW, et al. Incretin based drugs and the risk of pancreatic cancer: international multicentre cohort study. BMJ 2016;352:i581.

23. Chen H, Zhou X, Chen T, et al. Incretin-Based Therapy and Risk of Pancreatic Cancer in Patients with Type 2 Diabetes Mellitus: A Meta-analysis of Randomized Controlled Trials. Diabetes Ther 2016;7:725-42. 